\title{
FUNCTIONAL RESISTANCE TRAINING SUPERIORITY OVER CONVENTIONAL IN METABOLIC SYNDROME: A RANDOMIZED CLINICAL TRIAL
}

Turri-Silva, Natália'; Ricci-Vitor, Ana Laura ${ }^{3}$; Cipriano Jr, Gerson²; Garner, David ${ }^{4}$; Netto Jr, Jaime $^{5}$; Giacon, Thaís ${ }^{6}$; Christofaro, Diego ${ }^{7}$; Marques Vanderlei, Luiz Carlos ${ }^{8}$

${ }^{1}$ Natália Turri-Silva, Department of Physical Therapy, Faculty of Sciences and Technologies of UNESP \& Department of Physical Therapy and Physical Education, University of Brasilia. natalia.turri@hotmail.com

${ }^{2}$ Gerson Cipriano Jr, Department of Physical Therapy and Physical Education, University of Brasilia, Brasilia, Brazil. cipriano@unb.br

${ }^{3}$ Ana Laura Ricci-Vitor, Department of Physical Therapy, Faculty of Sciences and Technologies of UNESP, Presidente Prudente, São Paulo, Brazil analaura.ricci@yahoo.com.br

${ }^{4}$ David M. Garner, Department of Biological and Medical Sciences, Oxford Brookes University, Headington Campus, Gipsy Lane, Oxford OX3 0BP, United Kingdom. davidmgarner1@gmail.com

${ }^{5}$ Jaime Netto Jr, Department of Physical Therapy, Faculty of Sciences and Technologies of

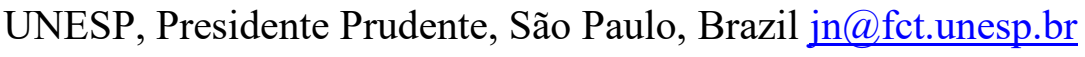

${ }^{6}$ Thais Roque Giacon, Department of Physical Therapy, Faculty of Sciences and Technologies of UNESP, Presidente Prudente, São Paulo, Brazil thaisgiacon@hotmail.com

${ }^{7}$ Diego Destro Christofaro, Department of Physical Therapy, Faculty of Sciences and Technologies of UNESP, Presidente Prudente, São Paulo, Brazil diego@,fct.unesp.br ${ }^{8}$ Luiz Carlos Marques Vanderlei, Department of Physical Therapy, Faculty of Sciences and Technologies of UNESP, Presidente Prudente, São Paulo, Brazil lcmvanderlei@fct.unesp.br

Corresponding author: Natália Turri da Silva

Affiliation where the research was conducted: Department of Physical Therapy, Faculty of Sciences and Technologies of UNESP 


\section{ABSTRACT}

Metabolic syndrome (MetS) is a growing epidemic related with higher values of blood pressure (BP) and autonomic dysfunction. Scientific evidence has been indicating that functional resistance training (FRT) is superior over conventional (CRT) for muscle fatigue and pain, yet its effects on autonomic modulation (AM), BP and heart rate in MetS are unclear.

We theorized that FRT can be superior to CRT in MetS patients because of larger muscle activation. This study compares FRT and CRT on AM, blood pressure, heart rate and muscle strength. Thirty-eight sex and age matched individuals (40 to 60 years) were randomized for FRT or CRT, with training intensity varying gradually from $30 \%-100 \%$ of one maximal repetition test (1MR), 3 times/week for 30 sessions. All outcomes were evaluated at baseline and post training. AM was assessed by heart rate variability (mean RR, RMSSD, SDNN, LF, HF, TINN, RRtri, SD1 and SD2). BP (mmHg) was obtained by cuff measures. Muscle strength was assessed by $1 \mathrm{MR}$. An increase in cardiac parasympathetic activity was observed in individuals allocated to FRT in comparison to CRT group (RMSSD $\Delta 40 \%$; SD1 $\Delta 39 \%$; and $\mathrm{HF} \mathrm{ms}^{2} \Delta 80 \%$ ). Moreover, just FRT was capable of reducing BP post intervention (SBP from $129.21 \pm 19.02$ to $118.94 \pm 14.14 \mathrm{mmHg}, \mathrm{p}<0.009, / \mathrm{d} /=0.49$; DBP from $85.26 \pm 11.48$ to $77.76 \pm 8.93 \mathrm{mmHg}, \mathrm{p}<0.01, / \mathrm{d} /=0.51)$. Both groups had a similar increase in muscle strength and no changes between HR. Progressive FRT was more beneficial to CRT regarding AM, increasing vagal activity, and reducing blood pressure in MetS individuals.

Keywords: metabolic syndrome; autonomic modulation; blood pressure; exercise therapy; resistance training. 


\section{Introduction}

Metabolic syndrome (MetS) is an increasing epidemic (O’Neill \& O'Driscoll, 2015), is the leading metabolic disease globally and one of the major factors in the development of heart failure (Perrone-Filardi et al., 2015). MetS is defined by the association of at least three risk factors amongst obesity, hyperglycemia, hypertension, low HDL cholesterol and hypertriglyceridemia (Alberti et al., 2009; Grundy et al., 2005). MetS has been associated with high levels of blood pressure (BP) (Licht, de Geus, \& Penninx, 2013) and autonomic dysfunction, with an increase in sympathetic activity (Stuckey, Tulppo, Kiviniemi, \& Petrella, 2014) impairing autonomic and hemodynamic control (de Carvalho et al., 2011). Also, impaired autonomic function has been highlighted as an underlying cause of MetS (Thorp \& Schlaich, 2018). Similarly, sympathetic activation was associated with left ventricular hypertrophy (Burns et al., 2007), further reinforcing the necessity for its control. Lifestyle modification, such as an exercise routine, has been emphasized to prevent and treat MetS, reducing cardiovascular risk (Kaur, 2014; Lemes et al., 2016; Wijndaele et al., 2007).

Previous studies have identified adverse links between muscle strength (Jurca et al., 2005; Wijndaele et al., 2007) or resistance exercise (Artero et al., 2012) and prevalence of MetS. Resistance training (RT) is an effective resource for cardiovascular risk populations with beneficial results on cardiac output, exercise capacity, glucose metabolism, fat mass and BP (Cordina et al., 2013; Strasser, Siebert, \& Schobersberger, 2010). Also, systematic review and meta-analysis has confirmed that progressive RT improves aerobic fitness in the same way as aerobic training for such populations, reinforcing its benefits (Hollings, Mavros, Freeston, \& Singh, 2017).

Amongst different RT treatment methods, progressive RT reaching high intensities seems to promote further benefits than moderate intensities, for example a greater increase in muscle strength (Bird, Tarpenning, \& Marino, 2005; Schoenfeld, Ogborn, \& Krieger, 2016), a 
positive effect on glycemic control in type 2 diabetes (Dunstan et al., 2002), better reduction of high density lipoprotein (HDL) and plasma fibrinogen levels (Sheikholeslami-Vatani, Ahmadi, Dehrashid, \& Gharibi, 2011), then improvement of cardiometabolic risk factors (Kemmler, Wittke, Bebenek, Fröhlich, \& Von Stengel, 2016). In consideration of those benefits, high intensity RT protocols seems an attractive method to treat individuals with MetS.

In view of training modalities, functional resistance training (FRT) has been applied besides conventional RT (CRT) (Pacheco, Teixeira, Franchini, \& Takito, 2013; Barbosa et al., 2016). FRT practices complex body movement levers by adopting specific postures that promote core instability and co-contractions, stimulating synergistic actions. FRT promoting chest instability through combinations of dynamic movements and isometric stabilization of the trunk (Pacheco et al., 2013; Barbosa et al., 2016; Phillips, 1997). By necessitating multi joint action, this modality appears to provide superior properties over muscle fatigue and soreness, which is predominantly important considering that pain can reduce statin effects in subjects with MetS under this treatment regime (Gentil, Fisher, \& Steele, 2017). Additionally, FRT causes lower cardiovascular responses during the training session in comparison to CRT (De Mello, Da Rosa, Vaz, \& Del Vecchio, 2017). FRT has already been studied in healthy women and offered benefits on autonomic modulation (AM) characterized by the increase on parasympathetic activity (Barbosa et al., 2016). From the available evidence, it therefore appears that FRT is a beneficial therapeutic approach for MetS treatment.

Still, the sympathetic overactivity in MetS can cause physical changes, harming baroreflex control (Limberg et al., 2014) decreasing blood flow in peripheral tissues and damaging endothelial function (Lind \& Lithell, 1993). "Regarding those factors, we hypothesized that FRT could better regulate the autonomic nervous system (ANS) and improve cardiovascular responses owing to activation of many muscle groups, promoting more neuromuscular and neurovascular stimuli, influencing the basic mechanism interactions linked to the autonomic and cardiovascular 
system. So, in terms of time, FRT could result in improved responses. Aiming to comprehend the consequences of RT modalities in MetS, this study compares the effects of progressive FRT and CRT on AM, blood pressure and heart rate $(\mathrm{HR})$ in patients with MetS.

\section{Methods}

\section{Study design}

This article presents data from a parallel randomized clinical trial, designed to determine whether different modalities of progressive RT enhance AM, BP, HR and muscle strength in individuals with MetS. Briefly, this was a 3-month protocol, with a total of 30 sessions of training, comparing a CRT to FRT interventions in individuals with MetS.

\section{Participants and setting}

Study participants were recruited in Presidente Prudente, São Paulo, Brazil, referred from physicians after recent clinical inspection (up until one year) to verify MetS according to the National Cholesterol Education Program-Adult Treatment Panel III (NCEP-ATP III) (Banerjee, 2005). The eligibility criteria included participants of both sex; age between 40 and 60 years old; who are not physically active in the last six months according to Physical Activity Guidelines for Americans, 2016 (Piercy et al., 2018); lacking reported respiratory and cardiovascular diseases; women before menopause; absence of cognitive dysfunction, without any inflammatory and infections process and orthopaedic injury; subjects who take medication for MetS risk factors alone. Also, all subjects included were referred by a physician after MetS diagnosis considering their blood tests (up to one year of validity). Patients who had problems in attending exercise sessions at the times available for research were excluded. Patients who discontinued intervention without stated reasons and those who were absent from the concluding evaluation were also excluded. 


\section{Written informed consent statement}

All participants signed a written informed consent statement contained the research proposal. The local ethics committee approved all procedures CAAE: 17378813.0.0000.5402.

\section{Randomization and allocation}

The technique used in this study was block randomization. This method ensures a balance for sample size across groups (the number of participants in each group was fixed between six to twelve, according to the availability of the participants over time). The result of randomization indicated the training modality of each participant (CRT or FRT). The random sequence of allocation, the application of the participants and the enrolment of participants were performed by a trained research physical therapist, who was blinded to the AM and cardiovascular system outcome evaluations.

The participants flow through different stages of the trial are reported in Figure 1 Figure 1 was organized according to the CONSORT statement (Schulz, Altman, \& Moher, 2010).

\section{Descriptive characteristic data}

The descriptive data collected for sample characterization were age, sex, mass, height, body mass index, heart rate, and the presence of risk factors for MetS (hypertension, obesity, diabetes, triglyceridemia, slow HDL).

\section{Test procedures and interventions}

Before the tests and the training protocol, there was a familiarization period with the training equipment. The patients were requested to graduate their efforts for this purpose after simulating 3 repetitions in each given load adjusted by the therapist. There were 5 options for 
the load perception: i) light, ii) light to moderate, iii) moderate, iv) moderate to intense, iv) intense. Familiarization and warm up consisted of 10 repetitions of each exercise load only if referred as light to moderate. The muscle groups emphasized for familiarization was the same as the training protocol.

Both training groups performed exercise training protocol three times per week. The subjects completed 30 training sessions. All participants completed all sessions. In case of a non-appearance the session was replaced.

Before each training session, there was a warm-up routine that consisted of walking for five minutes on a treadmill $(5 \mathrm{~km} / \mathrm{h})$ followed by a comprehensive stretching of muscles. The prescription including the dynamics of the exercise training (in terms of load, series and repetitions is thoroughly described in a previous study, but varied from $30 \%$ to $100 \%$ of 1 maximum repetition, 2 to 7 series, and 2 to 20 repetitions) (Turri-Silva et al., 2018). The time interval between groups was defined from 40 seconds until 90 seconds, according to equal time and load relationship (Bird et al., 2005). Muscle groups for both CRT and FRT were identical, only differing with regards to some postures from the training modality.

Eight exercises were completed in each training group. The exercises included on CRT protocol were: 1) bench press machine; 2) pectoral dorsal machine; 3) leg extensions machine; 4) leg curls machine; 5) leg press machine; 6) upright rows; 7) triceps pulley; 8) biceps pulley.

The exercises included on FRT were: 1') pectoral workout dorsal 45'; ') pectoral workout dorsal $45^{\circ}$ tilt; 3'), 4') and 5') were similar of conventional RT, 6') upright rows on Bozzu; 7') pushdowns dorsal $45^{\circ}$ tilt; 8') low-pulley curls dorsal $45^{\circ}$ tilt. All exercise movements included on FRT are illustrated in a previous study (Barbosa et al., 2016).

The exercises order in each training session followed from large to small muscle groups to maximize the total resistance lifted during the exercise bout. Two recuperative weeks 
were introduced alongside training protocol to secure expansion of muscle mass and optimize training effects.

\section{Primary outcomes}

\section{$\underline{\text { Heart rate variability }}$}

The primary outcome was the analysis of AM measured by heart rate variability (HRV), a simple, dependable, inexpensive and non-invasive method of monitoring ANS (Raimundo.,2015; Cam., 1996) and expressed via indices in the time and frequency domains. HRV measurements were achieved in both groups before and after training programs: on baseline moment and in the following week after the last training section.

HRV data acquisition was undertaken in the morning to avoid circadian changes. The room temperature was kept between $21^{\circ} \mathrm{C}$ and $23^{\circ} \mathrm{C}$ and relative air humidity between $40 \%$ and $60 \%$. Subjects were instructed to refrain from autonomic stimulants (alcoholic drinks, coffee, tea or food containing caffeine) for 24 hours preceding the evaluation, in addition to eating a light meal at least only 2 hours prior to measurement. The participants were instructed to maintain the use of their regular medications to control any cardiovascular risk factors. During the evaluation, the movement of people through the room was prohibited to reduce anxiety. Subjects were instructed to remain silent, awake, at rest, breathing spontaneously for 30 minutes in the supine position during the recording of the Polar RS800 monitor (Polar Electro $^{\circledR}$, Finland).

Only the temporal recordings containing at least $95 \%$ sino-atrial node beats were included in the study. Stationary frames of 1000 RR intervals (RRi) were selected, after excluding the first and last 5 minutes of data acquisition. The Kubios $H_{R V}{ }^{\circledR}$ software was necessary to complete HRV analysis (Biosignal Analysis and Medical Image Group, Department of Physics, University of Kuopio, Finland) (Tarvainen, Niskanen, Lipponen, 
Ranta-aho, \& Karjalainen, 2014).

HRV was measured in both time and frequency domains. For the time domain, the mean RR intervals (reflecting global variability) were used. The square root of the mean squared difference between adjacent RR intervals (RMSSD) indicating parasympathetic modulations of HR. The standard deviation of all normal RR intervals (SDNN) reflecting global variability. The geometric indexes from Poincaré plot (SD1 and SD2, indicating parasympathetic and global modulations of HR, respectively). Triangular interpolation of normal to normal RR intervals (TINN) and triangular index (RRtri) (De Carvalho et al., 2011; Vanderlei, Pastre, Hoshi, Carvalho, \& Godoy, 2009) both signifying global variability.

For the frequency domain, low frequency (LF: $0.04 \mathrm{~Hz}$ to $0.15 \mathrm{~Hz}$ ) and high frequency (HF: $0.15 \mathrm{~Hz}$ to $0.4 \mathrm{~Hz}$ ) were computed. Spectral analysis was computed using the Fast Fourier Transform (FFT) (Vanderlei et al., 2009). Spectral indexes were expressed in absolute units $\left(\mathrm{ms}^{2}\right)$ and normalized units (HF nu \& LF nu). The power in the LF band is modulated by both sympathetic and parasympathetic branches of the ANS and the power in the HF band is associated with vagal modulation (Electrophysiology, 1996).

\section{$\underline{\text { Blood Pressure and Heart Rate }}$}

Blood pressure (BP) was assessed using stethoscope and aneroid sphygmomanometer (Welch Allyn Durashock, USA) after at least 15 minutes at rest, followed by three measurements with 60 seconds of interval between them and considering the mean value, according to the recommendations from the American Heart Association (Pickering et al., 2005). As this method is not sensitive enough to detect deviations to 2 decimal places, all the measure of dispersion from statistical calculations were approximated to integers. HR was evaluated using a heart monitor (Polar Electro Oy ${ }^{\circledR}$, RS800, Finland). These measurements were completed for both groups on baseline moment and in the subsequent week after the last 
training section.

\section{Secondary outcomes}

\section{Muscle strength}

The one maximum repetition test (1RM) was completed to measure the maximum load in kilograms (Seo et al., 2012) and establish the individual loads of training to each movement according to training groups. This trial was likewise performed to allow the comparison of muscle strength between groups.

Before the 1 RM test there was a warming-up phase that consisted of walking for five minutes on a treadmill $(5 \mathrm{~km} / \mathrm{h})$ followed by a comprehensive stretching and 10 repetitions of resistance training for each modality with loads at light to moderate intensity, measured according to the patient's awareness as light, moderate or hard.

The 1 RM trial was commenced following the warming up period. The increment of loads happened according to the volunteer's perception. Just five attempts could be completed to establish the maximum load, but if it was necessary, the test could be scheduled for a new date to determine the precise loads. A three-minute interval of rest between the attempts to allow muscle recovery was allowed.

Muscle strength capacities were performed before starting the training protocol and until 72 hours after the termination of the training. The same evaluator applied all tests.

\section{Sample size}

The sample size was calculated considering the RMSSD index of HRV based on a previous study that compared comparable FRT and control groups in healthy individuals (Barbosa et al., 2016). In that study, RMSSD offered statistical significance for 13 healthy young participants (18 to 26 years). Bearing in mind the detected difference of $7 \mathrm{~ms}$ (Chang et 
al., 2010) at 5\% of significance level in a two-tailed hypothesis test, we ensured a test power of $80 \%$ with 19 participants per group.

\section{Statistical analysis}

The population was considered descriptively, mean and standard deviation values expressed the results, and through absolute and relative frequencies. Differences between descriptive data were confirmed using Shapiro-Wilk test followed by an Independent Student t-test for quantitative variables and the Fisher test for categorical variables.

Considering the outcomes -- as the assumptions of normality and homogeneity were not achieved through the Shapiro-Wilk test, non-parametric tests were applied. Intergroup comparisons (CRT or FRT) between the moments before and after training were evaluated through the Wilcoxon test. The intragroup differences (CRT or FRT) were evaluated via the delta values $(\Delta=$ after intervention - baseline) between the moments before and after training and compared by Mann-Whitney test. Differences were considered significant with $\mathrm{p}$ value $\leq$ 0.05. The effect size was calculated according to Cohen's $d$ algorithm (Cohen, 1988). Moreover, delta $(\Delta=$ post minus pre) were calculated to predict the value of changes in parasympathetic HRV indexes (RMSSD, HFms² and HFnu, SD1) on BP (SBP and DBP) in the FRT. After this, correlations (between $\triangle \mathrm{RMSSD}, \Delta \mathrm{HFms}^{2}, \Delta \mathrm{HFnu}, \triangle \mathrm{SD} 1$ vs. $\triangle \mathrm{SBP}$ and $\triangle \mathrm{DBP}$ ) were performed using the Spearman test. The extent of correlation is defined as $0.1<|\mathrm{r}|<0.3$ for small, $0.3<|\mathrm{r}|<0.5$ for moderate and $|\mathrm{r}|>0.5$ for strong. Linear regression was achieved for the variables that present significant differences for correlation. These statistical analyses were completed using the statistical software package SPSS v. 22 (SPSS, Inc. Chicago, USA) and GPower v. 3.1(Düsseldorf, Germany).

\section{Results}

Table 1 describes the sample description of the study. 
As indicated in Table 1, the intervention groups were similarly distributed in terms of their descriptive characteristic data.

Table 2 illustrates the intragroup comparisons of CRT and FRT between pre- and posttraining moments, and delta comparison between interventions for HRV variables, BMI and muscular strength.

As specified in Table 2, regarding the differences within groups, there is a statistically significant increase of parasympathetic activity (RMSSD, SD1) for FRT, while the decrease of global activity (SDNN, SD2) was specified for CRT. There was an rise in muscle strength for all muscle groups evaluated for both (FRT and CRT). In view of the differences between groups, there was an increase in RMSSD, SDNN, LF $\mathrm{ms}^{2}$ and $\mathrm{HF} \mathrm{ms}^{2}$ for FRT, but no changes in muscle strength.

Figure 2 describes intragroup comparisons for blood pressure and heart rate.

As shown by Figure 2, there was a statistical difference between moments pre- and post-FRT intervention for systolic and diastolic blood pressure (SBP and DBP). The SBP was reduced from $129 \pm 19$ to $119 \pm 14 \mathrm{mmHg}(\Delta:-10 \pm 5 \mathrm{mmHg})$ following an effect size of 0.4944 considered moderately large according to Cohen's $d$ interpretation and DBP reduced from 85 \pm 11 to $78 \pm 9 \mathrm{mmHg}(\Delta:-7 \pm 2 \mathrm{mmHg})$ following an effect size of 0.5181 considered as large according to Cohen's $d$ interpretation. Regarding HR data there was a deterioration for the FRT group (from $73 \pm 11$ to $69 \pm 11 \mathrm{bpm} ; \Delta:-4 \pm 11 \mathrm{bpm}$ ) but without statistical significance.

Outside the results above, there was an inverse and moderate correlation between $\Delta$ HFnu vs. $\Delta$ SBP $(\mathrm{p}=0.014, \mathrm{r}=-0.555)$ and between $\Delta$ HFnu vs. $\Delta \mathrm{DBP}(\mathrm{p}=0.020, \mathrm{r}=-0.530)$. The added indexes $\left(\Delta \mathrm{RMSSD}, \Delta \mathrm{SD} 1, \Delta \mathrm{HFms}^{2}\right)$ presented no significant correlation. Hereafter, the prediction equation was set as $\Delta \mathrm{SBP}=-7.657+(-0.835 \times \Delta \mathrm{HFnu})$. Indicating that $\Delta \mathrm{HFnu}$ statistically predicted $\triangle \mathrm{SBP}$, accountable for $28.40 \%$ of the variation $(\mathrm{p}=0.019)$. Another quantity $\Delta \mathrm{HFnu}$ leads to a $0.835,(95 \% \mathrm{CI},-1.513$ to -0.157$)$ responsible for a decrease in $\Delta \mathrm{SBP}$. 
The hypothesized equation for $\Delta \mathrm{DBP}$ was $\Delta \mathrm{DPB}=585.920+(1676.580 \times \Delta \mathrm{HFnu})$. Indicating, $\triangle \mathrm{HFnu}$ statistically significantly predicted $\triangle \mathrm{DBP}$, accountable for $25.90 \%$ of the variation $(\mathrm{p}=0.026)$. A further quantity $\Delta \mathrm{HFnu}$ leads to a $0.617,(95 \% \mathrm{CI},-1.152$ to -0.083$)$ responsible for a decrease in $\triangle \mathrm{DBP}$.

\section{Discussion}

This study advocates that FRT results in better AM than CRT, providing enhanced responses to the parasympathetic branch of ANS and reducing BP. This is an original study comparing effects of resistance training modalities in participants with MetS in addition to BP and AM by using linear indexes of HRV. The results suggest that progressive FRT until attaining high intensities of training $(100 \% 1 \mathrm{RM})$ is an attractive modality to treat autonomic dysfunction and pressure control in MetS individuals. There were no changes between both modalities regarding muscle strength gain.

This study permits a better understanding of ANS components separately and its effects over cardiovascular parameters, supplementing findings from a previous study (TurriSilva et al., 2018). Earlier, our research group discovered that FRT and CRT appears to increase chaotic responses, specifically the nonlinear dynamics of HRV, by observing a new non-linear HRV analysis technique (Turri- Silva et al., 2018). This study complements the understanding of ANS branches influencing BP and HR.

The parasympathetic increase after FRT is a significant result, as vagal activation helps to regulate intrinsic autonomic unbalance in MetS, categorized by higher sympathetic branch activation of the ANS (Stuckey et al., 2014). A reduction of global autonomic variability in CRT group was expressed by lower values of SDNN and SD2 indexes. We observe this reduction could be sporadic if the small parasympathetic variation is considered between pre- and post-CRT, which can have influenced the decrease in global variability. So, no effects 
on AM after CRT may be specified. Previous review articles have demonstrated no changes in HRV after CRT in different populations such as young, old, healthy, hypertensive or individuals with fibromyalgia (Kingsley \& Figueroa, 2016). Recently, Trevizani and colleagues presented no deviations of HRV indexes after CRT in hypertensive subjects (Trevizani et al., 2018).

In contrast, FRT achieved higher global variability in comparison to CRT, expressed by delta values, with a further RMSSD increase. Improving autonomic dysfunction in MetS is pivotal as the ANS modulates all visceral functions and, HRV impairment reflects a functional and early pointer of cardiac injury (Tulppo, Makkikkallio, Seppannen, Laukkanen, \& Huikuri, 1998). Increasing parasympathetic modulation in autonomic dysfunction can be beneficial as an anti-fibrillation effect, improvements on left ventricle function and mitigation of systemic inflammation, already reported by studies exploring parasympathetic stimulation in myocardial infarction and heart failure ( $\mathrm{He}, \mathrm{Lu}, \mathrm{He}, \mathrm{Huang}, \&$ Jiang, 2016). Our proposal is a similar physiological effect because of vagal increase in FRT, providing cardiovascular protection in MetS, although the latter study was completed in patients with cardiovascular diseases.

A possible elucidation for the improved results after FRT is the larger muscle mass activation on this modality (Matos-Santos, 2017). A former study reported individuals with MetS presented preserved functional sympatholysis and higher exercise blood flow during dynamic forearm exercise (Limberg et al., 2014). Thus, FRT may have promoted higher blood flow during the training sections, resulting in better chronic response, influencing autonomic regulation. Similarly, the improvements on AM after FRT may be attributed to an increase in baroreceptor reflex activity, as demonstrated by the growth trend of LF power in comparison to CRT (Goldstein et al., 2011; Rahman, Pechnik, Gross, Sewell, \& DS, 2011). 
Regarding HR effects, there was no statistical change between groups, yet FRT presented lower results following training. Furthermore, in relation to BP effects, changes on SBP were observed only on the FRT group, differing from literature reviews indicating that RT reduces around 4.1 mmHg of SBP (Lemes et al., 2016). With regards the mean values, we discovered a decrease of $10 \mathrm{mmHg}$ for SBP and around $7 \mathrm{mmHg}$ to DBP on FRT intragroup comparison. Reductions of these amounts are considered clinically pertinent since it can alter the hypertension stage of hypertensive participants (Goldstein et al., 2011). This is specifically relevant as hypertension is considered one of the principal MetS risk factors (Banerjee, 2005). Decreases greater than $2 \mathrm{mmHg}$ in BP could lead to a reduction of approximately $10 \%$ of mortality regarding the cerebral vascular accidents and 7\% related to cardiovascular diseases (Lewington, Clarke, Qizilbash, Peto, \& Collins, 2002).

Improved effects on BP after FRT could similarly be related to the higher number of active muscle groups on this modality, inducing better responses on peripheral circulation because of an increase in oxygen consumption (Duling, \& Klitzman, 1980). A previous study reported substantial BP reduction in women with MetS after an acute resistance exercise session (Tibana, Boullosa, Leicht, \& Prest, 2013) which may support our findings. The nonappearance of a BP reduction in CRT can be ascribed to the lower baseline values in this group, which was lower than under normotensive settings.

We examined if the parasympathetic increase could have impacted BP reduction in FRT. Our results exposed that parasympathetic increase post-FRT was accountable for a decrease in SBP of $28.40 \%$ and DBP of $25.90 \%$. This result demonstrated that further mechanisms may have inclined the BP reduction. Besides ANS, numerous other mechanisms can influence BP (Hall et al., 2012) for instance hormonal changes on renin-angiotensinaldosterone system and natriuretic factor (Chopra, Baby, \& Jacob, 2011). Similarly, local tissue control modifications (nitric oxide, endothelin, inflammatory cytokines, oxygen reactive 
species) (Chopra et al., 2011; Hall et al., 2012) and renal mechanisms (glomerular filtration) (Hall et al., 2012) can alter BP control.

Both training exercises increased muscle strength equally as expected after RT protocols (Chodzko-Zajko et al., 2009; Schoenfeld et al, 2016). Muscle strength is a vital outcome for participants with MetS, largely to optimize glucose transportation (Richter, \& Hargreaves, 2013). The authors distinguished some limitations, such as the absence of a control group, the lack of technological resources to perform hormonal, renal and local tissue substances analysis which limited the understanding of physiological mechanisms involved. Correspondingly, the authors did not apply the Borg scale to quantify the subjective perception of effort during familiarization yet the load was controlled by a therapist with rehabilitation expertise. Nevertheless, despite those limitations, the current study offers a unique perspective in an under-represented population, and the first study to compare resistance training protocols on linear HRV indexes of AM, HR and BP control in MetS. This study promotes a better understanding of the scientific and therapeutic values, providing superior evidence for a possible clinical technique. In conclusion, progressive FRT presented superior benefits over CRT in MetS, by increasing vagal activity and reducing BP. There were no alterations between FRT and CRT for HR and muscle strength.

\section{ACKNOWLEGMENT}

The authors would like to thank the Laboratory of Stress Physiology and LAFIDE members from the Department of Physical Therapy, Faculty of Sciences and Technologies of UNESP for their support during this study.

\section{FUNDING}

This work was supported by "Fundação de Amparo à Pesquisa do Estado de São Paulo" (FAPESP) under Grant (Process: 2015/10229-0). 


\section{REFERENCES}

Alberti, K. G. M. M., Eckel, R. H., Grundy, S. M., Zimmet, P. Z., Cleeman, J. I., Donato, K. A., ... Smith, S. C. (2009). Harmonizing the metabolic syndrome: A joint interim statement of the international diabetes federation task force on epidemiology and prevention; National heart, lung, and blood institute; American heart association; World heart federation; International. Circulation, 120(16), 1640-1645.

https://doi.org/10.1161/CIRCULATIONAHA.109.192644

Artero, E. G., Lee, D., Lavie, C. J., España-Romero, V., Sui, X., Church, T. S., \& Blair, S. N. (2012). Effects of muscular strength on cardiovascular risk factors and prognosis. $J$ Cardiopulm Rehabil, 32(6), 351-358.

https://doi.org/10.1097/HCR.0b013e3182642688.Effects

Bird, S. P., Tarpenning, K. M., \& Marino, F. E. (2005). Designing resistance training programmes to enchance fitness: A review of the acute programme. Sports Medicine, 35(10), 841-851. https://doi.org/10.2165/00007256-200535100-00002

Burns, J., Sivananthan, M. U., Ball, S. G., Chir, M. B. B., Mackintosh, A. F., Mary, D. A. S. G., ... John, P. (2007). The Relationship Between Central Sympathe DRIVE AND MRI DETERMINED LEFT VENTRICULAR MASS IN ESSENTIAL HYPERTENSION, 115(15), 1999-2005. https://doi.org/10.1161/CIRCULATIONAHA.106.668863.THE

Chang, J. S., Yoo, C. S., Yi, S. H., Hong, K. H., Lee, Y. S., Oh, H. S., ... Ahn, Y. M. (2010). Changes in heart rate dynamics of patients with schizophrenia treated with risperidone. Progress in Neuro-Psychopharmacology and Biological Psychiatry, 34(6), 388-396. 
https://doi.org/10.1016/j.pnpbp.2010.04.017

Chodzko-Zajko, W. J., Proctor, D. N., Fiatarone Singh, M. A., Minson, C. T., Nigg, C. R., Salem, G. J., \& Skinner, J. S. (2009). Exercise and physical activity for older adults. Medicine and Science in Sports and Exercise, 41(7), 1510-1530.

https://doi.org/10.1249/MSS.0b013e3181a0c95c

Chopra, S., Baby, C., \& Jacob, J. J. (2011). Neuro-endocrine regulation of blood pressure. Indian Journal of Endocrinology and Metabolism. https://doi.org/10.4103/2230-8210.86860

Cohen, J. (1988). Statistical Power Analysis for the Behavioral Sciences. (P. Press, Ed.) (2ns ed.). New York, NY.

Cordina, R. L., O’Meagher, S., Karmali, A., Rae, C. L., Liess, C., Kemp, G. J., ... elermajer, D. S. (2013). Resistance training improves cardiac output, exercise capacity and tolerance to positive airway pressure in Fontan physiology.International Journal of Cardiology, 168(2), 780-788. https://doi.org/10.1016/j.ijcard.2012.10.012

de Carvalho, T. D., Marcelo Pastre, C., Claudino Rossi, R., de Abreuc, L. C., Valenti, V. E., \& Marques Vanderleia, L. C. (2011). Geometric Indices of Heart Rate Variability in Chronic Obstructive Pulmonary Disease. Portuguese Journal of Pulmonology, 17(6), 260-265. https://doi.org/10.1016/j.rppneu.2011.06.007

de Mello, T. L., da Rosa, S. M., Vaz, M. dos S., \& Del Vecchio, F. B. (2017). Strength training in session with polyarticular exercises generates inferior cardiovascular stress than 
the training session with monoarticular exercises. Brazilian Journal of Sports Sciences, 39(2), 132-140. https://doi.org/10.1016/j.rbce.2016.02.006

Duling, B. K. B. (1980). of Microvascular Function : Ann. Rev. Physiol, 42(1), 373-382.

Dunstan, D. W., Daly, R. M., Owen, N., Jolley, D., de Courten, M., Shaw, J., \& Zimmet, P. (2002). High-Intensity Resistance Training Improves Glycemic Control in Older Patients With Type 2 Diabetes. Diabetes Care, 25(10), 1729-1736. https://doi.org/10.2337/diacare.25.10.1729

Electrophysiology, T. F. of the E. S. of C. the N. A. S. of P. (1996). Heart Rate Variability Standards of Measurement, Physiological Interpretation, and Clinical Use. Circulation, 93(1), 1043-1065. https://doi.org/https://doi.org/10.1161/01.CIR.93.5.1043

Gentil, P., Fisher, J., \& Steele, J. (2017). A Review of the Acute Effects and Long-Term Adaptations of Single- and Multi-Joint Exercises during Resistance Training. Sports Medicine, 47(5), 843-855. https://doi.org/10.1007/s40279-016-0627-5

Goldstein, L. B., Bushnell, C. D., Adams, R. J., Appel, L. J., Braun, L. T., Chaturvedi, S., ... Pearson, T. A. (2011). Guidelines for the primary prevention of stroke: A Guideline for Healthcare Professionals from the American Heart Association/American Stroke Association. Stroke, 42(2), 517-584. https://doi.org/10.1161/STR.0b013e3181fcb238

Grundy, S. M., Cleeman, J. I., Daniels, S. R., Donato, K. A., Eckel, R. H., Franklin, B. A., ... Costa, F. (2005). Diagnosis and management of the metabolic syndrome: An American Heart 
Association/National Heart, Lung, and Blood Institute scientific statement. Circulation, 112(17), 2735-2752. https://doi.org/10.1161/CIRCULATIONAHA.105.169404

Hall, J. E., Granger, J. P., do Carmo, J. M., da Silva, A. A., Dubinion, J., George, E., ... Hall, M. E. (2012). Hypertension: Physiology and pathophysiology. Comprehensive Physiology, 2(4), 2393-2442. https://doi.org/10.1002/cphy.c110058

He, B., Lu, Z., He, W., Huang, B., \& Jiang, H. (2016). Autonomic Modulation by Electrical Stimulation of the Parasympathetic Nervous System: An Emerging Intervention for Cardiovascular Diseases. Cardiovascular Therapeutics, 34(3), 167-171. https://doi.org/10.1111/1755-5922.12179

Hollings, M., Mavros, Y., Freeston, J., \& Fiatarone Singh, M. (2017). The effect of progressive resistance training on aerobic fitness and strength in adults with coronary heart disease: A systematic review and meta-analysis of randomised controlled trials. European Journal of Preventive Cardiology, 24(12), 1242-1259. https://doi.org/10.1177/2047487317713329

Jurca, R., Lamonte, M. J., Barlow, C. E., Kampert, J. B., Church, T. S., \& Blair, S. N. (2005). Association of muscular strength with incidence of metabolic syndrome in men. Medicine and Science in Sports and Exercise, 37(11), 1849-1855. https://doi.org/10.1249/01.mss.0000175865.17614.74

Kemmler, W., Wittke, A., Bebenek, M., Fröhlich, M., \& Von Stengel, S. (2016). High intensity resistance training methods with and without protein supplementation to fight 
cardiometabolic risk in middle-aged males: A randomized controlled trial. BioMed Research International, 2016. https://doi.org/10.1155/2016/9705287

Kingsley, J. D., \& Figueroa, A. (2016). Acute and training effects of resistance exercise on heart rate variability. Clinical Physiology and Functional Imaging, 36(3), 179- 187. https://doi.org/10.1111/cpf.12223

Lemes, Í. R., Ferreira, P. H., Linares, S. N., MacHado, A. F., Pastre, C. M., \& Netto, J. (2016). Resistance training reduces systolic blood pressure in metabolic syndrome: A systematic review and meta-analysis of randomised controlled trials. British Journal of Sports Medicine, 50(23), 1438-1442. https://doi.org/10.1136/bjsports- 2015-094715

Lewington, S., Clarke, R., Qizilbash, N., Peto, R., \& Collins, R. (2002). Age-specific relevance of usual blood pressure to vascular mortality: a meta-analysis of individual data for one million adults in 61 prospective studies.Age-specific relevance of usual blood pressure to vascular mortality: a meta-analysis of individual d. Lancet, 360(9349), 1903-1913.

Licht, C. M. M., de Geus, E. J. C., \& Penninx, B. W. J. H. (2013). Dysregulation of the Autonomic Nervous System Predicts the Development of the Metabolic Syndrome. The Journal of Clinical Endocrinology \& Metabolism, 98(6), 2484-2493. https://doi.org/10.1210/jc.2012-3104

Limberg, J. K., Morgan, B. J., Sebranek, J. J., Proctor, L. T., Eldridge, M. W., \& Schrage, W. G. (2014). Neural control of blood flow during exercise in human metabolic syndrome. Experimental Physiology, 99(9), 1191-1202. https://doi.org/10.1113/expphysiol.2014.078048 
Lind, L., \& Lithell, H. (1993). Decreased peripheral blood flow in the pathogenesis of the metabolic syndrome comprising hypertension, hyperlipidemia, and hyperinsulinemia. American Heart Journal, 125(5 PART 2), 1494-1497. https://doi.org/10.1016/00028703(93)90446-G

O’Neill, S., \& O’Driscoll, L. (2015). Metabolic syndrome: A closer look at the growing epidemic and its associated pathologies. Obesity Reviews, 16(1), 1-12. https://doi.org/10.1111/obr.12229

Pacheco, M. M., Teixeira, L. A. C., Franchini, E., \& Takito, M. Y. (2013). Functional Vs. Strength Training in Adults: Specific Needs Define the Best Intervention. International Journal of Sports Physical Therapy, 8(1), 34-43.

Perrone-Filardi, P., Paolillo, S., Costanzo, P., Savarese, G., Trimarco, B., \& Bonow, R. O. (2015). The role of metabolic syndrome in heart failure. European Heart Journal, 36(39), 2630-2634. https://doi.org/10.1093/eurheartj/ehv350

Pickering, T. G., Hall, J. E., Appel, L. J., Falkner, B. E., Graves, J., Hill, M. N., ... Roccella, E. J. (2005). Recommendations for Blood Pressure Measurement in Humans and Experimental Animals: Part 1: Blood Pressure Measurement in Humans: A Statement for Professionals From the Subcommittee of Professional and Public Education of the American Heart Association Cou. Hypertension, 45(1), 142-161. https://doi.org/10.1161/01.HYP.0000150859.47929.8e 
Piercy KL, Troiano RP, Ballard RM, Carlson SA, Fulton JA, Galuska DA, ... Olson RD. The

Physical Activity Guidelines for Americans. JAMA. 2018;320(19):2020-2028. doi:10.1001/jama.2018.14854

Rahman, F., Pechnik, S., Gross, D., Sewell, L., \& DS, G. (2011). LF Power Reflects Baroreflex Function, Not Cardiac Sympathetic Innervation. Clinical Autonomic Research, 21(3), 133141. https://doi.org/10.1007/s10286-010-0098-y.LF

Rezende Barbosa, M. P. da C. de, Netto Júnior, J., Cassemiro, B. M., de Souza, N. M., Bernardo, A. F. B., da Silva, A. K. F., ... Vanderlei, L. C. M. (2016). Impact of functional training on cardiac autonomic modulation, cardiopulmonary parameters and quality of life in healthy women. Clinical Physiology and Functional Imaging, 36(4), 318-325. https://doi.org/10.1111/cpf.12235

Richter, E. A., \& Hargreaves, M. (2013). Exercise, GLUT4, and Skeletal Muscle Glucose Uptake. Physiological Reviews, 93(3), 993-1017.

https://doi.org/10.1152/physrev.00038.2012

Schoenfeld, B. J., Ogborn, D., \& Krieger, J. W. (2016). Effects of Resistance Training Frequency on Measures of Muscle Hypertrophy: A Systematic Review and Meta- Analysis. Sports Medicine, 46(11), 1689-1697. https://doi.org/10.1007/s40279-016-0543-8

Schulz, K. F., Altman, D. G., \& Moher, D. (2010). CONSORT 2010 Statement: updated guidelines for reporting parallel group randomised trials. BMJ, 340(mar23 1), c332-c332. https://doi.org/10.1136/bmj.c332 
Seo, D. Il, Kim, E., Fahs, C. A., Rossow, L., Young, K., Ferguson, S. L., ... So, W. Y. (2012). Reliability of the one-repetition maximum test based on muscle group and sex. Journal of Sports Science and Medicine, 11(2), 221-225. https://doi.org/10.1590/S151786922003000500007

Sheikholeslami-Vatani, D; Ahmadi, S; Dehrashid,KA; Gharibi, F. (2011). Changes in cardiovascular risk factors and inflammatory markers of young, healthy, men after six weeks of moderate or high intensity resistance training. The Journal of Sports Medicine and Physical Fitness, 51(4), 695-700.

Strasser, B., Siebert, U., \& Schobersberger, W. (2010). Resistance Training in the Treatment of the Metabolic Syndrome: A Systematic Review and Meta-Analysis of the Effect of Resistance Training on Metabolic Clustering in Patients with Abnormal Glucose Metabolism. Sports Medicine, 40(5), 397-415. https://doi.org/10.2165/11531380-000000000-00000

Stuckey, M. I., Tulppo, M. P., Kiviniemi, A. M., \& Petrella, R. J. (2014). Heart rate variability and the metabolic syndrome: A systematic review of the literature.

Diabetes/Metabolism Research and Reviews, 30(8), 784-793.

https://doi.org/10.1002/dmrr.2555

Sujit Banerjee. (2005). Final Report. Public Health, (02), 1-161.

https://doi.org/10.2172/875800

Tarvainen, M. P., Niskanen, J. P., Lipponen, J. A., Ranta-aho, P. O., \& Karjalainen, P. A. 
(2014). Kubios HRV - Heart rate variability analysis software. Computer Methods and Programs in Biomedicine, 113(1), 210-220. https://doi.org/10.1016/j.cmpb.2013.07.024

Thorp, A. A., \& Schlaich, M. P. (2018). Relevance of Sympathetic Nervous System Activation in Obesity and Metabolic Syndrome, 2015. Retrieved from https://www.hindawi.com/journals/jdr/2015/341583/

Tibana, R. A., Boullosa, D. A., Leicht, A. S., \& Prestes, J. (2013). Women with metabolic syndrome present different autonomic modulation and blood pressure response to an acute resistance exercise session compared with women without metabolic syndrome. Clinical Physiology and Functional Imaging, 33(5), 364- 372. https://doi.org/10.1111/cpf.12038

Trevizani, G. A., Seixas, M. B., Benchimol-Barbosa, P. R., Vianna, J. M., da Silva, L. P., \& Nadal, J. (2018). Effect of Resistance Training on Blood Pressure and Autonomic Responses in Treated Hypertensives. Journal of Strength and Conditioning Research, 32(5), 1462-1470. https://doi.org/10.1519/JSC.0000000000001995

Tulppo, MP; Makkikallop, TH; Laukkanen, RT; Huikuri, H. (1998). Vagal modulation of heart rate during exercise: effects of age and physical fitness. Am. J. Physiol, 274(Heart Circ. Physiol. 43), H424-H429.

Turri-Silva, N., Garner, D. M., Moosavi, S. H., Ricci-Vitor, A. L., Christofaro, D. G. D., Netto Junior, J., ... Vanderlei, L. C. M. (2018). Effects of resistance training protocols on nonlinear analysis of heart rate variability in metabolic syndrome. Brazilian Journal of Medical and Biological Research = Revista Brasileira de Pesquisas Medicas e Biologicas, 
51(8), e7459. https://doi.org/10.1590/1414- 431X20187459

Vanderlei, L. C. M., Pastre, C. M., Hoshi, R. A., Carvalho, T. D. De, \& Godoy, M. F. De. (2009). Basic notions of heart rate variability and its clinical applicability. Revista Brasileira de Cirurgia Cardiovascular : Orgao Oficial Da Sociedade Brasileira de Cirurgia Cardiovascular, 24(2), 205-217. https://doi.org/10.1590/S0102-76382009000200018

Wijndaele, K., Duvigneaud, N., Matton, L., Duquet, W., Thomis, M., Beunen, G., ... Philippaerts, R. M. (2007). Muscular strength, aerobic fitness, and metabolic syndrome risk in flemish adults. Medicine and Science in Sports and Exercise, 39(2), 233-240. https://doi.org/10.1249/01.mss.0000247003.32589.a6 


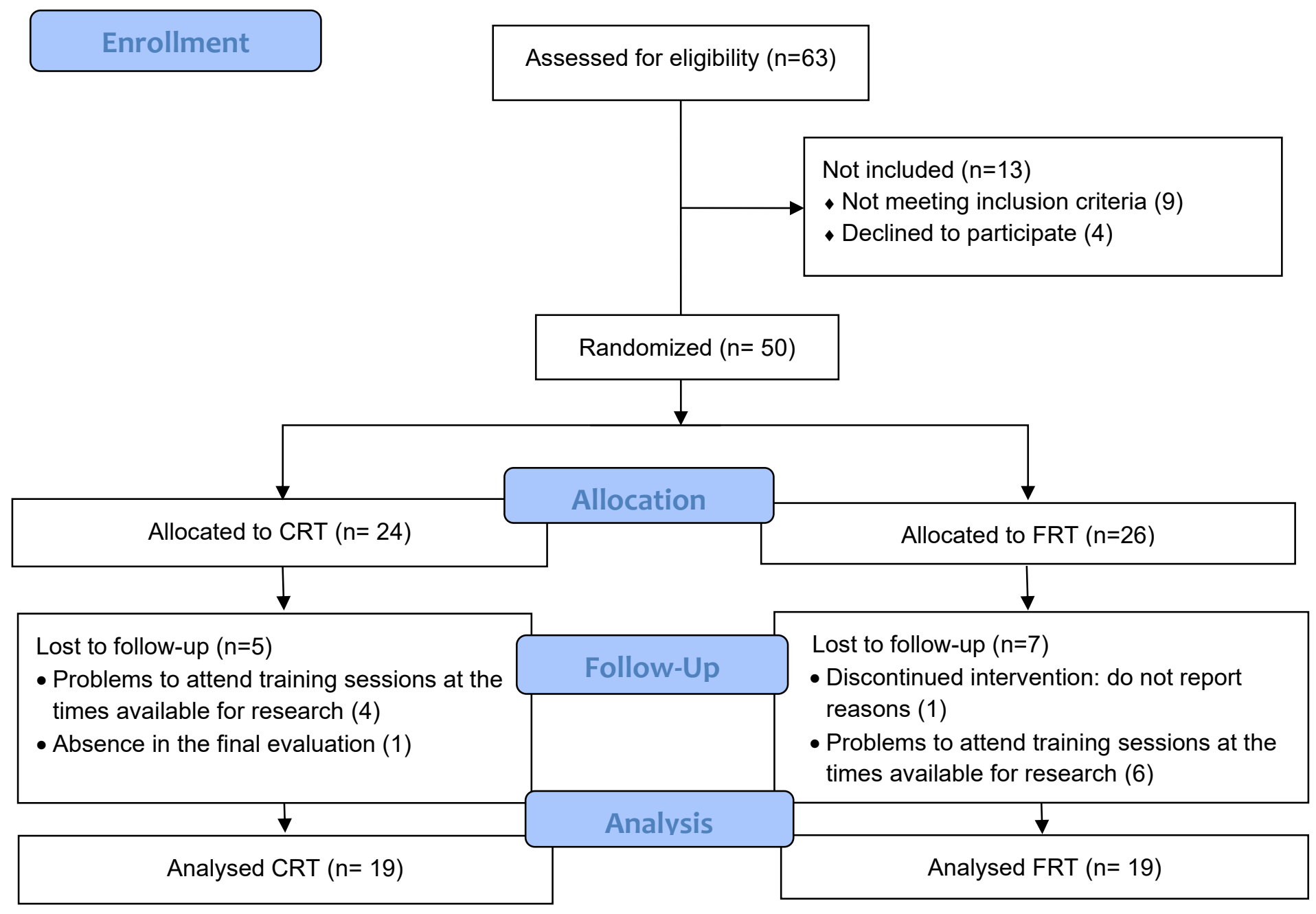

Figure 1. Consort Flow Diagram of participants with metabolic syndrome who underwent conventional resistance training (CRT) and functional resistance training (FRT) 

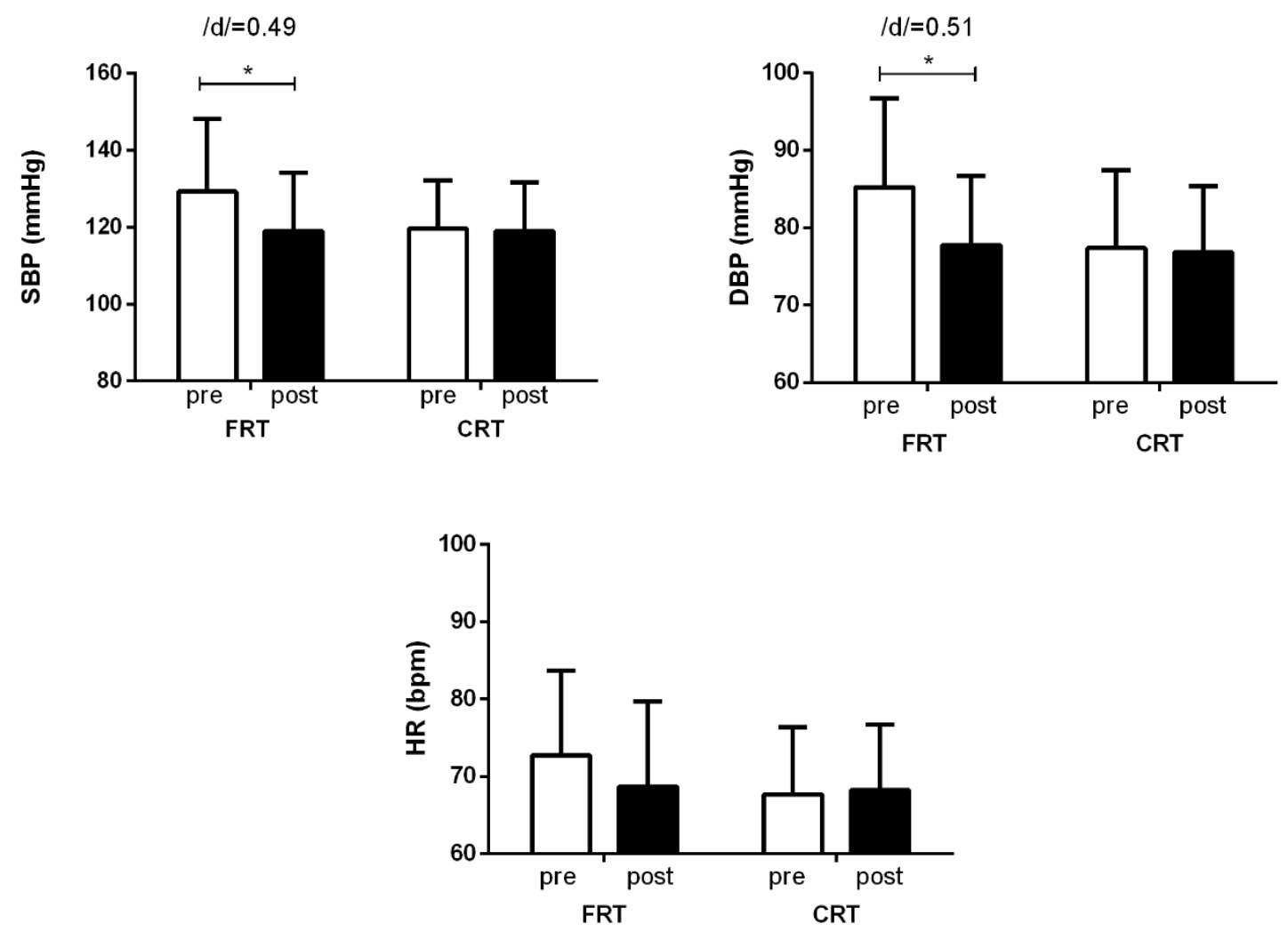

Figure 2. Intragroup comparisons between pre and post training moments for systolic and diastolic blood pressure (SBP and DBP, respectively) and heart rate (HR) for MetS participants of functional resistance training (FRT) and conventional resistance training (CRT). * Statistical significance $(\mathrm{p} \leq 0.05)$ by Student t-test; /d/ effect size by Cohen's $d$. 
Table 1. Metabolic syndrome sample characterization between training groups.

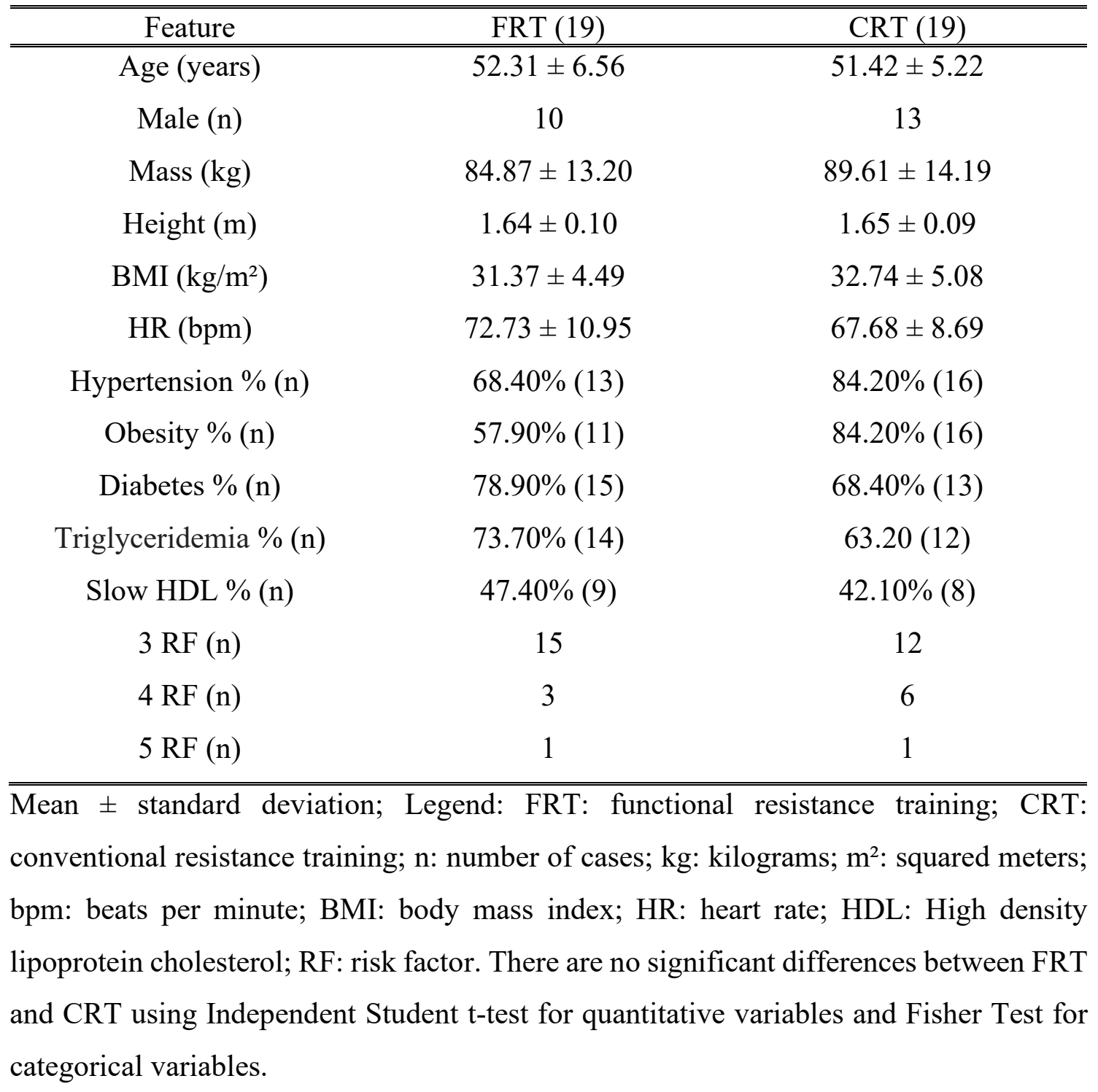


Table 2. Intragroup and intergroup comparisons of metabolic syndrome individuals following FRT and CRT

\begin{tabular}{|c|c|c|c|c|c|c|}
\hline \multirow{2}{*}{$\begin{array}{c}\text { Variables } \\
\text { HRV }\end{array}$} & \multicolumn{2}{|c|}{ FRT } & \multicolumn{2}{|c|}{ CRT } & \multicolumn{2}{|c|}{$\Delta$ Comparisons } \\
\hline & Before & After & Before & After & $\Delta \mathrm{FRT}$ & $\Delta \mathrm{CRT}$ \\
\hline Mean RR (ms) & $844.90(131.30)$ & $909.60(239.30)$ & $925.60(126.80)$ & $899.50(149.60)$ & $41.60(132.60)$ & $-4.70(58.40)$ \\
\hline RMSSD (ms) & $15.60(11.40)$ & $21.90(26.60) *$ & $22.00(13.60)$ & $21.70(14.40)$ & $2.30(11.00)$ & $0.00(10.00) \dagger$ \\
\hline TINN (ms) & $155.00(60.00)$ & $125.00(95.00)$ & $150.00(130.00)$ & $130.00(100.00)$ & $-15.00(55.00)$ & $0.00(105.00)$ \\
\hline RRtri & $9.43(6.18)$ & $11.09(5.27)$ & $10.52(5.96)$ & $9.01(3.41)$ & $0(3.27)$ & $-0.20(3.14)$ \\
\hline SD1 (ms) & $11.10(8.10)$ & $15.50(18.80)^{*}$ & $15.60(9.60)$ & $15.30(10.20)$ & $1.80(7.90)$ & $0.00(7.10)$ \\
\hline LF (nu) & $73.70(23.20)$ & $69.00(21.10)$ & $73.40(29.20)$ & $67.10(30.20)$ & $-2.60(11.10)$ & $0.50(15.20)$ \\
\hline $\mathrm{HF}\left(\mathrm{ms}^{2}\right)$ & $86.00(128.00)$ & $155.00(416.00)$ & $172.00(213.00)$ & $144.00(214.00)$ & $50.00(185.00)$ & $-32.00(132.00) \dagger$ \\
\hline HF (nu) & $26.30(23.10)$ & $31.10(21.10)$ & $26.60(29.20)$ & $32.90(28.80)$ & $2.60(11.10)$ & $-0.50(15.30)$ \\
\hline \multicolumn{7}{|l|}{ 1RM (kg) } \\
\hline Pectoralis & $35.00(41.25)$ & $47.50(37.50)^{*}$ & $45.00(35.00)$ & $47.50(46.25)^{*}$ & $-5.00(15.00)$ & $-10.00(16.25)$ \\
\hline Back & $27.50(30.00)$ & $38.50(23.75)^{*}$ & $40.00(20.00)$ & $45(21.25)^{*}$ & $7.50(10.75)$ & $5.00(10.00)$ \\
\hline Leg Press & $98.50(66.50)$ & $123.00(73.80) *$ & $109.00(38.50)$ & $140.50(59.50) *$ & $26.50(22.25)$ & $28(29.75)$ \\
\hline
\end{tabular}

Median (interquartile range) *Significant differences by Wilcoxon test between intragroup comparisons; $\uparrow$ Significant differences by Mann Whitney test between intergroup delta comparisons; $p<0,05$. Legend: $H R V=$ heart rate variability; Mean $R R=$ mean of $R R$ intervals; $R M S S D=$ the root mean square of successive difference between normal intervals consecutive cardiac beats; SDNN= the standard deviation of normal-to-normal intervals; TINN= triangular interpolation of the interval between consecutive heart beats; RRtri= triangular index; SD1= standard deviation of instant variability beat to beat; $\mathrm{SD} 2=$ standard deviation of long-term interval between consecutive heart beats; $\mathrm{LF}=$ low frequency; $\mathrm{HF}=$ high frequency; $\mathrm{ms}=$ milliseconds; $\mathrm{nu}=$ normalized units; $\mathrm{kg}=$ kilograms; $1 \mathrm{RM}=$ one repetition maximum; $\mathrm{FRT}=$ functional resistance training, $\mathrm{CRT}=\mathrm{conventional}$ resistance training. 